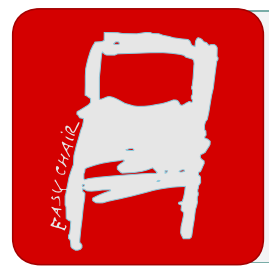

EPiC Series in Health Sciences

Volume 4, 2020, Pages 28-34

CAOS 2020. The 20th Annual Meeting of the International Society for Computer Assisted Orthopaedic Surgery

\title{
Predicting Cementless Stem Fixation in Total Hip Arthroplasty Using Radiodensity Mapping
}

\author{
Teja Chrukuri ${ }^{1}$, Chameka Madurawe ${ }^{1}$. Jim Pierrepont ${ }^{1}$, Jonathan Baré ${ }^{2}$, \\ Stephen McMahon ${ }^{3}$ and Andrew Shimmin ${ }^{2}$ \\ ${ }^{1}$ Corin, Cirencester, U.K \\ ${ }^{2}$ Melbourne Orthopaedic Group, VIC, Australia \\ ${ }^{2}$ Malabar Orthopaedic Clinic, VIC, Australia
}

\begin{abstract}
Femoral component loosening due to poor fixation is a common failure mode in cementless total hip arthroplasty (THA). We sought to develop a technique to predicted femoral stem fixation using pre-operative $\mathrm{CT}$.

Forty-nine patients requiring THA received pre-operative CTs as part of Corin OPS $^{\mathrm{TM}} 3 \mathrm{D}$ dynamic planning. All patients received a taper wedged blade stem $\left(\mathrm{TriFit}^{\mathrm{TM}}\right.$ TS, Corin UK) implanted through a posterior approach. Post-operatively, patients received an immediate CT and AP x-ray and a 1-year follow-up x-ray. The distance between the most superior point of the greater trochanter and the shoulder of the stem was measured and scaled using the known cup diameter on both the immediate and 1year follow-up x-rays. The difference was recorded as stem subsidence. Subsidence greater than $4 \mathrm{~mm}$ was deemed clinically relevant. The precise placement of the stem determined by registering the known 3D implant geometry to the post-op CT was virtually implanted back into the pre-operative OPS ${ }^{\mathrm{TM}}$ planning software. A colour map of the bone density at the stem/bone interface using the Hounsfield Units (HU) of each CT pixel was generated. Blue represents low density bone transitioning through to green and then red.

Mean stem subsidence was $1.8 \mathrm{~mm}$ (0 to $11.1 \mathrm{~mm}$ ). Five patients had clinically relevant subsidence. Significant areas of blue and green around the proximal portion of the stem was observed in high subsidence stems when compared to the stems with minimal subsidence.

Using the HU of the CT scan as an indicator for bone density, we were able to predict poor implant fixation and subsequent subsidence in a taper wedge stem.
\end{abstract}




\section{Introduction}

Total Hip Arthroplasty (THA) is a highly successful and a common surgical procedure to restore function and reduce pain [1]. Over the years there have been a significant advance in prosthesis design ranging from the shape, coating and modality of fixation [2]. However, post-operative complications due to mechanical failure of implants still occur and component loosening is considered as one of the most common failure modes in cementless THA [3-5]. Although the risk of femoral loosening can be minimised by optimal component positioning, other factors such as patient age, weight, gender, osteopenia, stem design and Dorr-C bone have all been proposed as risk factors for poor fixation and subsequent stem subsidence and poor outcome [6-9].

Bone density analysis from Computer Tomography (CT) imaging is defined as means value expressed in Hounsfield Units (HU) in each pixel of the CT and is currently being used to study bone quality and its relationship for femoral fracture [10,11]. These analyses have created clinical opportunities for the use of CT imaging and bone density to analyse the correlation between component fixation and post-operative implant subsidence in THA. With the increased popularity of CT-based assistive technologies in THA, in this study we sought to develop a technique to predict femoral stem fixation using pre-operative CT.

\section{Method}

Fourty-nine patients requiring THA were randomly selected from a previous study investigating component alignment. Mean age was 66 (51 to 82), and 41\% were female. All patients received preoperative CT as part of OPSTM 3D dynamic planning. Intra-operatively all patients received a taper wedged blade stem (TriFit ${ }^{\mathrm{TM}}$, Corin UK) and a cemented cup (Trinity ${ }^{\mathrm{TM}}$, Corin UK) implanted through posterior approach. Post-operatively, patients received an immediate CT and AP x-ray prior to leaving the hospital, and a 1-year follow-up x-ray.

On both the immediate post-op x-ray and 1-year follow-up x-ray, the known cup diameter was used to scale the image. On both images, the distance between the most superior point of the greater trochanter and the shoulder of the stem was measured. The difference was recorded as stem subsidence. Subsidence greater than $4 \mathrm{~mm}$ was deemed clinically relevant.

The post-operative CT was used to determine the precise three-dimensional placement of the stem immediately after surgery by registering the known 3D implant geometry to the CT. For each patient, the achieved stem position from post-op CT was then virtually implanted back into the pre-operative OPS $^{\mathrm{TM}}$ planning software. The software provides a colour map of the bone density at the stem/bone interface using the $\mathrm{HU}$ of each pixel of the CT. Blue represents low density bone transitioning through to green and then red (most dense).

\section{Results}

Mean stem subsidence was $1.8 \mathrm{~mm}$ (0 to $11.1 \mathrm{~mm}$ ). Five patients had clinically relevant subsidence:

- $68 \mathrm{M}$ subsided $11.1 \mathrm{~mm}$

- $58 \mathrm{M}$ subsided $5.0 \mathrm{~mm}$

- $63 \mathrm{~F}$ subsided $4.9 \mathrm{~mm}$

- $55 \mathrm{M}$ subsided $4.7 \mathrm{~mm}$

- $66 \mathrm{M}$ subsided $4.3 \mathrm{~mm}$ 
Radiodensity colour plots for all five patients with clinically relevant subsidence had significant areas of blue (low density bone) around the proximal portion of the stem, with minimal medium/high density fixation when compared to the stems with minimal subsidence (Fig 1).

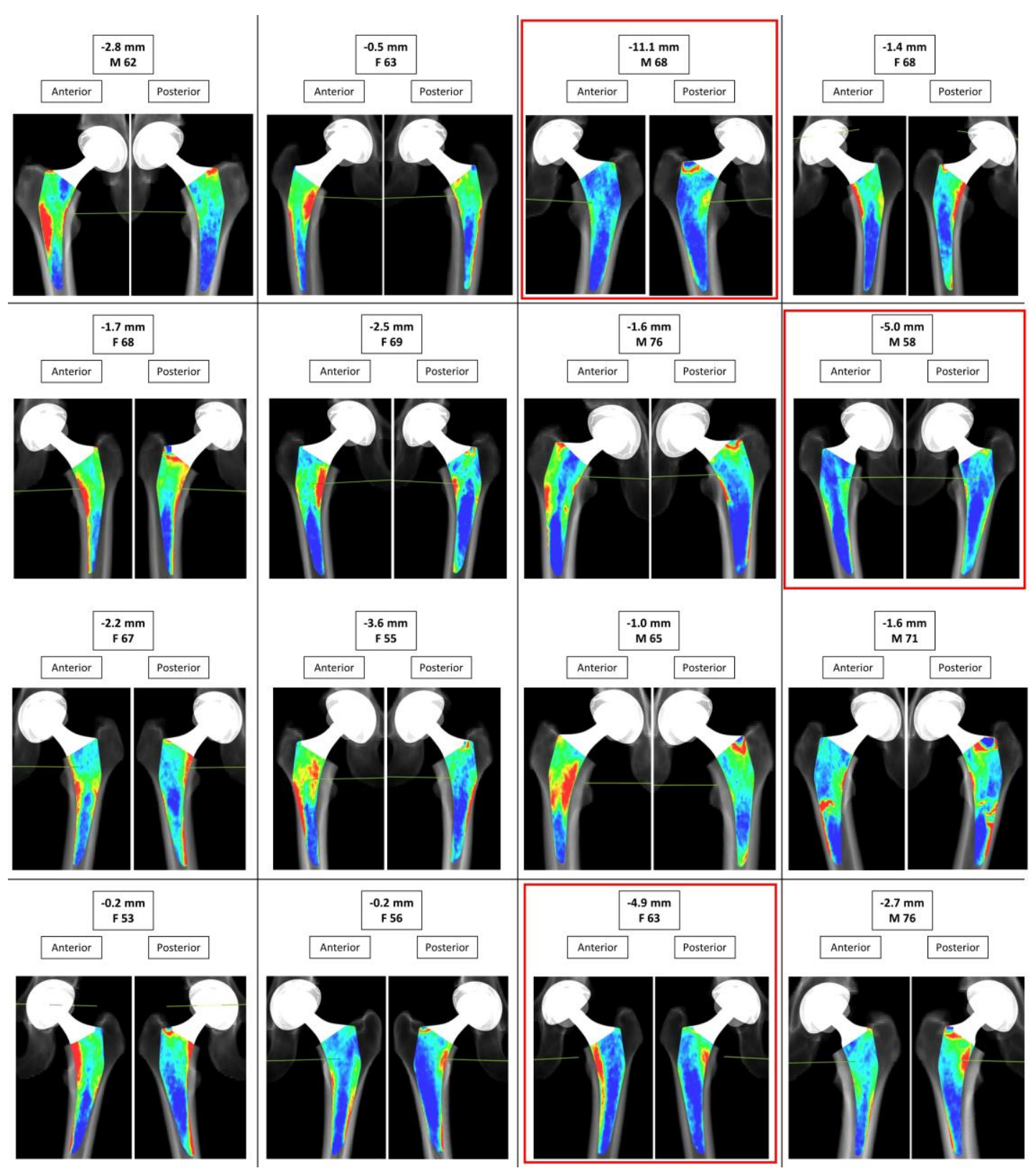




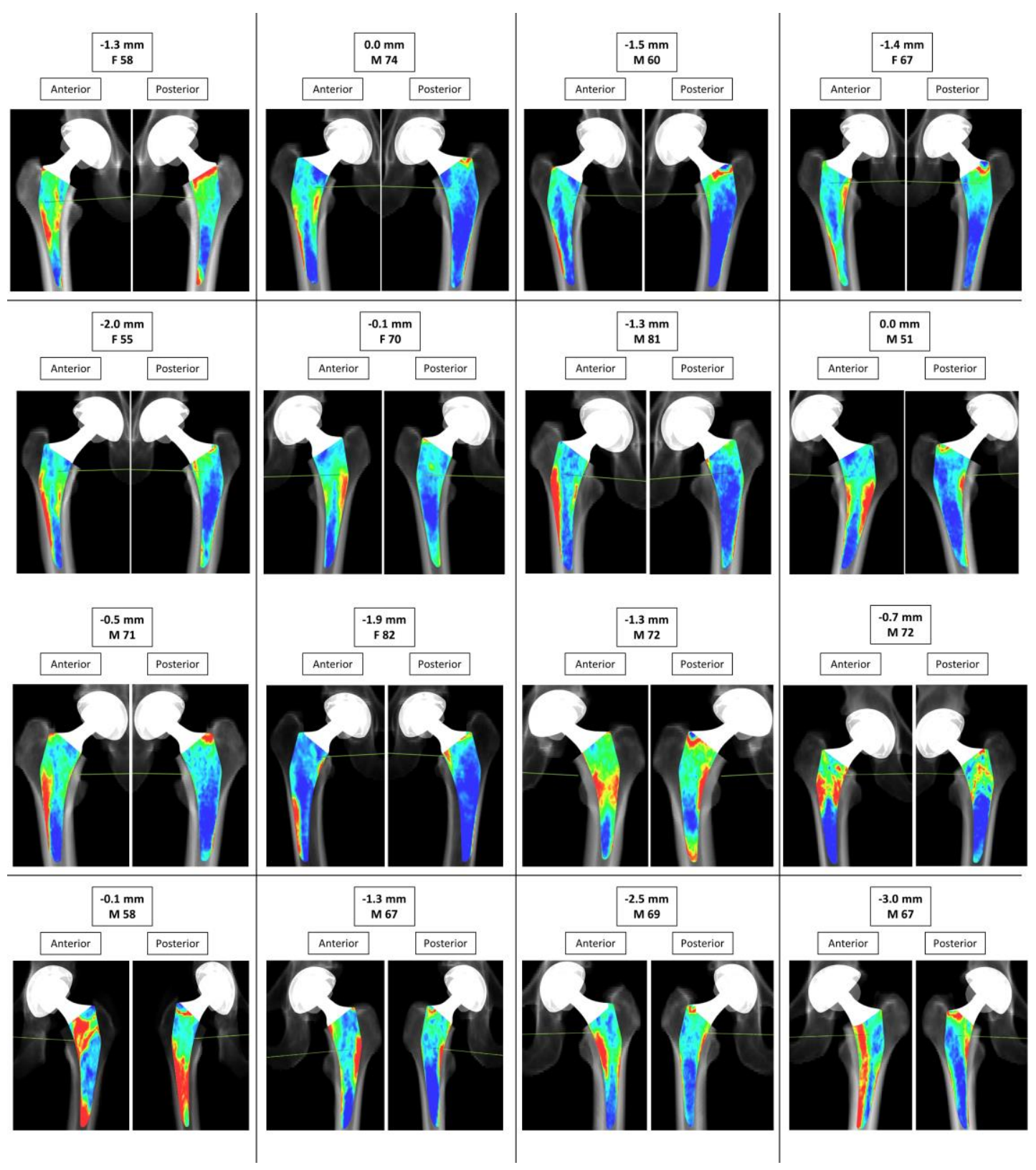




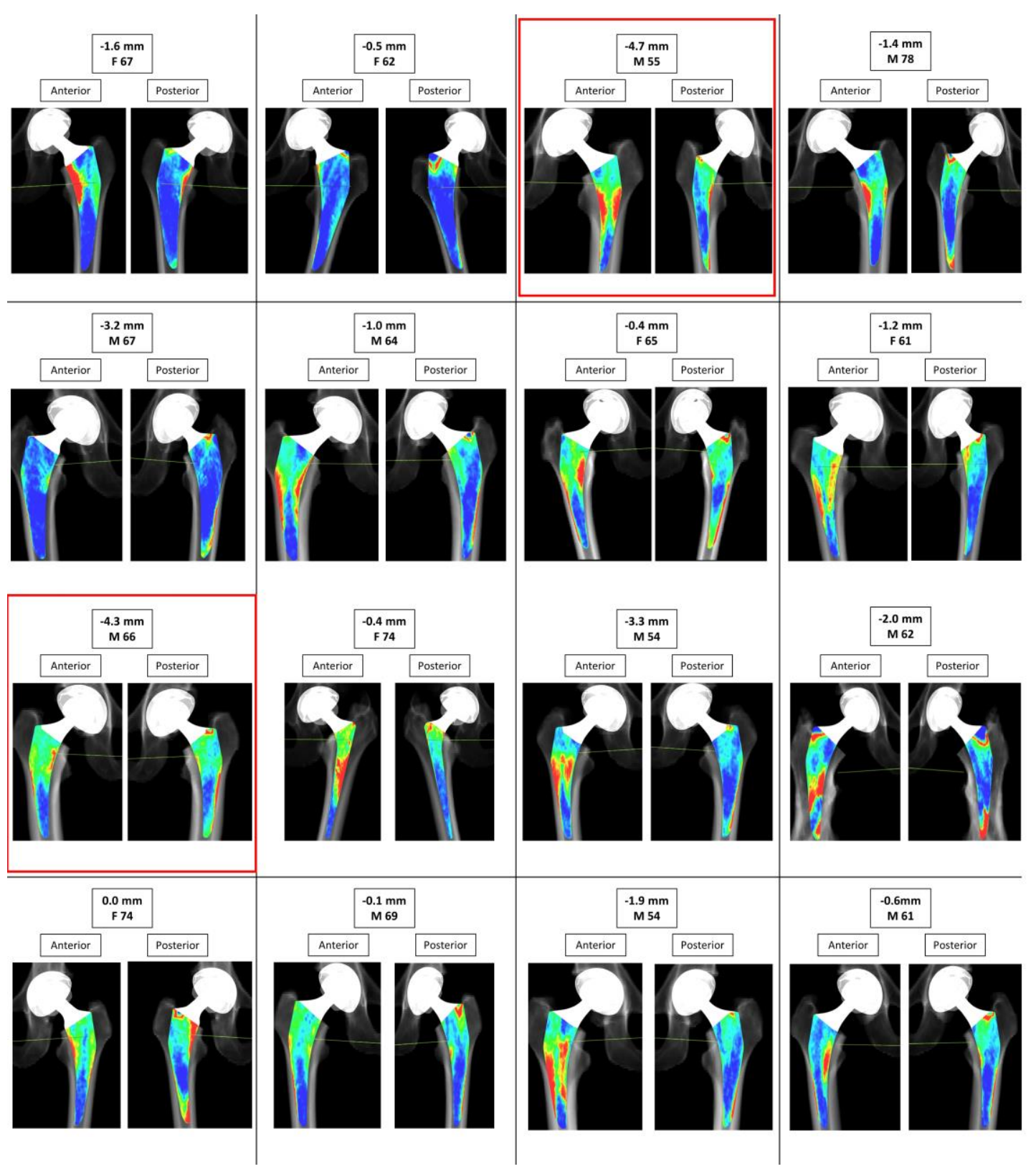




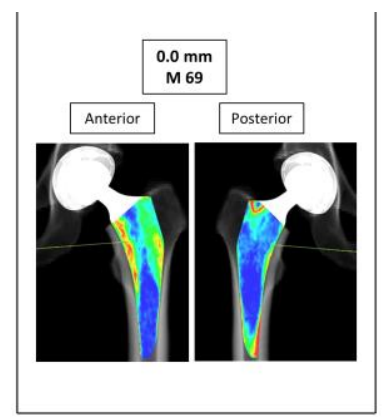

Figure 1: Radiodensity mapping results for implant fixation

\section{Discussion}

Although advanced implant designs feeds into the success of Total Hip Replacement surgery, post-operative complications due to mechanical failure of implants still occur. Femoral stem loosening due to poor fixation is considered as one the most common failure modes in cementless THA. Using the Hounsfield units of the CT scan as an indicator for bone radiodensity, we were able to predict poor implant fixation and subsequent subsidence in a taper wedge stem. This technique can be used as a fixation guide during pre-operative implant size and position templating to ensure optimal post-operative implant fixation.

Radiodensity mapping is a well-established analysis method to study bone quality. Previous studies have been conducted on correlation between bone quality, femoral fracture and subsidence utilising CTs [10-11]. A study conducted by Inoue et al aiming to quantify the implant-femur contact state and develop a technique to analyse the initial fixation pattern of cementless femoral stem using density mapping investigated the effect of varus/valgus of the stem positioning in cortical contact. Their results indicated that quantifying the contact state of implant and femur by using radiodensity mapping is a useful technique to accurately analyze the fixation pattern of a cementless femoral stem [12].

Through this study we have developed a technique to predict femoral stem fixation using radiodensity mapping from pre-operative CT. However, when determining the correlation of the radiodensity distribution to stem subsidence factors effecting subsidence was not considered. Further analysis is required to quantify the predicted implant fixation from radiodensity mapping in consideration with operative factors (implant size and femoral offset) and patient specific parameters (BMI and bone quality) effecting implant subsidence.

\section{Conclusion}

This new technology might have pre-operative value in providing a more quantitative measure of fixation and resultant stem choice. Future studies are planned to investigate the effect of stem design and area of fixation in post-operative subsidence and fracture. 


\section{References}

1. Garellick, G., Malchau, H., Herberts, P., Hansson, E., Axelsson, H. and Hansson, T., 1998. Life expectancy and cost utility after total hip replacement. Clinical orthopaedics and related research, (346), pp.141-151.

2. Zagra, L., 2017. Advances in hip arthroplasty surgery: what is justified? EFORT open reviews, 2(5), pp.171-178.

3. Novikov, D., Mercuri, J.J., Schwarzkopf, R., Long, W.J., Bosco III, J.A. and Vigdorchik, J.M., 2019. Can some early revision total hip arthroplasties be avoided? The bone \& joint journal, 101(6_Supple_B), pp.97-103.

4. Gwam, C.U., Mistry, J.B., Mohamed, N.S., Thomas, M., Bigart, K.C., Mont, M.A. and Delanois, R.E., 2017. Current epidemiology of revision total hip arthroplasty in the United States: National Inpatient Sample 2009 to 2013. The Journal of arthroplasty, 32(7), pp.2088-2092.

5. Sadoghi, P., Liebensteiner, M., Agreiter, M., Leithner, A., Boehler, N. and Labek, G., 2013. Revision surgery after total joint arthroplasty: a complication-based analysis using worldwide arthroplasty registers. The Journal of arthroplasty, 28(8), pp.13291332.

6. Al-Najjim, M., Khattak, U., Sim, J. and Chambers, I., 2016. Differences in subsidence rate between alternative designs of a commonly used uncemented femoral stem. Journal of orthopaedics, 13(4), pp.322-326.

7. Wyatt, M., Frampton, C. and Hooper, G., 2017. Is stem design important in uncemented total hip replacement to decrease subsidence in obese patients?. Annals of Joint, 2(50), pp.1-4.

8. Ries, C., Boese, C.K., Dietrich, F., Miehlke, W. and Heisel, C., 2019. Femoral stem subsidence in cementless total hip arthroplasty: a retrospective single-centre study. International orthopaedics, 43(2), pp.307-314.

9. Faisal, M., Thomas, G. and Young, S.K., 2011. Subsidence of the Corail femoral component in the elderly. A retrospective radiological review. Hip International, 21(3), pp.325-329.

10. Vivanco, J.F., Burgers, T.A., García-Rodríguez, S., Crookshank, M., Kunz, M., MacIntyre, N.J., Harrison, M.M., Bryant, J.T., Sellens, R.W. and Ploeg, H.L., 2014. Estimating the density of femoral head trabecular bone from hip fracture patients using computed tomography scan data. Proceedings of the Institution of Mechanical Engineers, Part H: Journal of Engineering in Medicine, 228(6), pp.616-626.

11. Pétursson, P., Edmunds, K.J., Gíslason, M.K., Magnússon, B., Magnúsdóttir, G., Halldórsson, G., Jónsson, H. and Gargiulo, P., 2015. Bone mineral density and fracture risk assessment to optimize prosthesis selection in total hip replacement. Computational and mathematical methods in medicine, 2015.

12. Inoue, D., Kabata, T., Maeda, T., Kajino, Y., Fujita, K., Hasegawa, K., Yamamoto, T., Takagi, T., Ohmori, T. and Tsuchiya, H., 2015. Usefullness of three-dimensional templating software to quantify the contact state between implant and femur in total hip arthroplasty. European Journal of Orthopaedic Surgery \&

Traumatology, 25(8), pp.1293-1300. 